\title{
COATING AND MANDREL EFFECTS ON FABRICATION OF GLOW DISCHARGE POLYMER NIF SCALE INDIRECT DRIVE CAPSULES
}

by
A. NIKROO, J.M. PONTELANDOLFO, and E.R. CASTILLO 


\section{DISCLAIMER}

This report was prepared as an account of work sponsored by an agency of the United States Government. Neither the United States Government nor any agency thereof, nor any of their employees, makes any warranty, express or implied, or assumes any legal liability or responsibility for the accuracy, completeness, or usefulness of any information, apparatus, product, or process disclosed, or represents that its use would not infringe privately owned rights. Reference herein to any specific commercial product, process, or service by trade name, trademark, manufacturer, or otherwise, does not necessarily constitute or imply its endorsement, recommendation, or favoring by the United States Government or any agency thereof. The views and opinions of authors expressed herein do not necessarily state or reflect those of the United States Government or any agency thereof. 


\title{
COATING AND MANDREL EFFECTS ON FABRICATION OF GLOW DISCHARGE POLYMER NIF SCALE INDIRECT DRIVE CAPSULES
}

\author{
by \\ A. NIKROO, J.M. PONTELANDOLFO, and E.R. CASTILLO
}

This is a preprint of a paper to be presented at the 14th Target Fabrication Meeting, July 15-19, 2001, West Point, New York and to be published in Fusion Technology.

\author{
Work supported by \\ the U.S. Department of Energy \\ under Contract No. DE-AC03-01SF22260
}

GA PROJECT 30095

APRIL 2002 


\title{
COATING AND MANDREL EFFECTS ON FABRICATION OF GLOW DISCHARGE POLYMER NIF SCALE INDIRECT DRIVE CAPSULES
}

\author{
A. Nikroo, J.M. Pontelandolfo, and E.R. Castillo \\ Inertial Fusion Division \\ General Atomics, P.O. Box 85608, San Diego, California 92186-5608
}

(858) 455-2931, email: abbas.nikroo@gat.com

\begin{abstract}
Targets for the National Ignition Facility (NIF) need to be about $200 \mu \mathrm{m}$ thick and $2 \mathrm{~mm}$ in diameter. These dimensions are well beyond those currently fabricated on a routine basis. We have investigated fabrication of near NIF scale targets using the depolymerizable mandrel technique. Poly-alpha-methylstyrene (PAMS) mandrels, about $2 \mathrm{~mm}$ in diameter, of varying qualities were coated with as much as $125 \mu \mathrm{m}$ of glow discharge polymer (GDP). The surface finish of the final shells was examined using a variety of techniques. A clear dependence of the modal spectrum of final GDP shell on the quality of the initial PAMS mandrels was observed. Isolated features were found to be the greatest cause for a shell not meeting the NIF standard.
\end{abstract}

\section{INTRODUCTION}

The goal of the National Ignition Facility (NIF) being built in the U.S. is to achieve ignition in a laboratory setting. A major step in realization of this goal is the fabrication of a suitable target. There are a number of different designs for targets needed for ignition. For indirect drive laser fusion, they all involve a thick walled $(>150 \mu \mathrm{m})$ capsule roughly $2 \mathrm{~mm}$ in diameter. The surface finish of an ignition target needs to meet certain stringent criteria. Currently, an ideal modal power spectrum, shown in Fig. 1, is used in ignition simulations and is referred to as the NIF standard. This power spectrum indicates the allowed magnitude of perturbations of different wavelengths, or mode numbers, on the surface of the shell. In particular, mode 2 corresponds to nonsphericity, while modes $>100$ refer to the background surface finish.

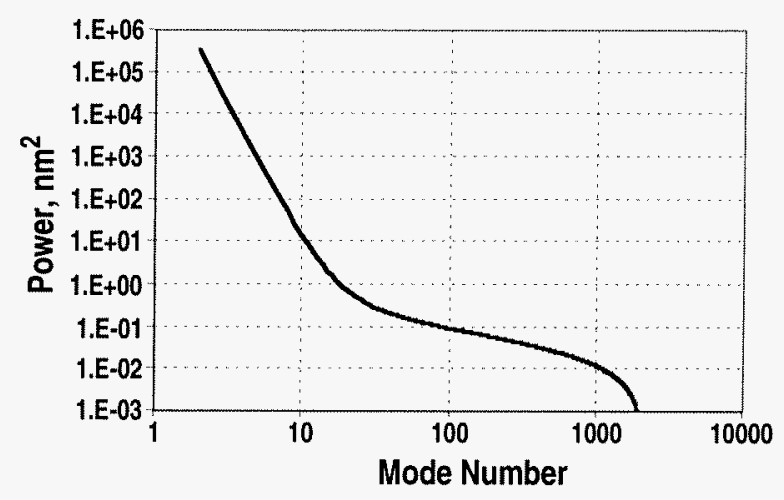

Fig. 1. Current modal power spectrum used for NIF shell designs and simulations.

The GDP deposition process has been a key step in fabrication of Inertial Confinement Fusion (ICF) capsules for a number of years. Many of the current laser fusion capsules are entirely made up of GDP or at least contain a GDP layer. In fact, the power spectrum shown in Fig. 1 is an average of the best ten GDP coated shells shot on the now decommissioned NOVA laser facility. In one of the current NIF capsule designs the capsule is entirely made up of GDP (possibly doped with a small amount of high Z elements). GDP shells are also currently being used as mandrels for vapor deposited polyimide and beryllium. Therefore, GDP capsules are likely to continue to play an important role in target fabrication and achieving ignition on NIF.

The bulk of the previous work has concentrated on smaller $(0.4$ and $1 \mathrm{~mm}$ diameter $)$ and thinner $(<50 \mu \mathrm{m})$ shells. This paper discusses extending the previous work to thicker and larger shells required for indirect drive ignition. The current status of production of $2 \mathrm{~mm}$ GDP 
shells, both thinner walled $(\sim 15 \mu \mathrm{m})$ as mandrels for overcoating with polyimide, and thick walled (>100 $\mu \mathrm{m})$ GDP shells and the major problems remaining are discussed. In particular, the effects of imperfections present on the starting mandrels or caused by the GDP coating process on the surface finish of GDP shells are discussed.

\section{EXPERIMENTAL}

Currently, most ICF capsules are made using the depolymerizable mandrel technique ${ }^{1}$ using PAMS mandrels. We used this technique in our study as well. The details of the technique as used for ICF target fabrication can be found in the literature. ${ }^{2}$ The depolymerizable mandrel technique allows unparalleled control of wall thickness and composition. Each step of this seemingly simple process has been the subject of extensive development over the past few years. The quality of the final capsule depends heavily on the quality of the product of each step. The final GDP shell will usually mimic the PAMS mandrel. The starting PAMS mandrel must therefore have the sphericity, concentricity and surface finish required of ICF targets. The GDP coating must be deposited in a controlled fashion to obtain the desired thickness and surface finish of the final capsule. If dopants are desired in the ablator, they may be added to the GDP film in the desired concentrations. Finally, the pyrolysis conditions have to be chosen to ensure survival of capsules during this process. The final dimensions of capsules are also influenced by the pyrolysis parameters.

GDP coatings were performed using a helical resonator plasma system which has been described elsewhere. ${ }^{3}$ This system offers a number of unique advantages required for making laser fusion targets. By prudent selection of coating parameters, coatings as thick as $50 \mu \mathrm{m}$ can be routinely deposited with very small amounts of residual stress and excellent surface finish. Precise control of the coating rate allows precise control of the wall thickness of shells. In short, high quality ICF capsules have been made using this technique for over a decade.

For this study freshly cleaved mica flats were used as substrates, as well as 1 to $2 \mathrm{~mm}$ diameter PAMS shells. The mica flats provided ultra-clean substrates, which were practically defect free and atomically smooth. Two types of $2 \mathrm{~mm}$ diameter PAMS shells were used for this study. One type was made using polyacrylic acid (PAA), ${ }^{4}$ which we denote as PAA-PAMS. The other type was made using polyvinyl alcohol (PVA), denoted PVA-PAMS here. The $1 \mathrm{~mm}$ shells used for this work were all of the PVAPAMS type. Shells were agitated either with a piezo shaker or with a tapping system, both of which are described elsewhere. ${ }^{5}$ The piezo shaker usually works best for smaller shells (1 mm or smaller in diameter) and was used for this work when coating $1 \mathrm{~mm}$ shells. The tapping system was used for coating $2 \mathrm{~mm}$ diameter shells. The quality of the final GDP shells will depend on both the quality of the PAMS mandrel and the GDP coating. Flaws in the PAMS shells are directly reproduced in the GDP shells. The shells used for this work, in general, very nearly or completely satisfied the NIF standard modal spectrum.

We examined the coatings and the shells using a number of different techniques. The background surface finish was measured by atomic force microscopy (AFM) on small patches of shells. Scanning Electron Microscopy (SEM) was used to examine the surface for domes and high frequency roughness. Coating thickness was measured destructively using SEM on shell cross-sections and non-destructively by interferometry. AFM spheremaps were used to obtain the modal spectra, which are currently the ultimate measure of shell quality. 6

\section{RESULTS AND DISCUSSION}

Initially, a number of coating runs was made on mica substrates. The coating thicknesses were over $100 \mu \mathrm{m}$. Figure 2 shows an AFM patch scan of such a coating. As can be seen the background roughness measured $(2.2 \mathrm{~nm})$ is well within that desired for NIF. These experiments showed that the GDP coating parameters were suitable for thick coatings.

We next used $1 \mathrm{~mm}$ PAMS shells as substrates. These shells were agitated using the piezo bouncer. A relatively small number of shells (25) were coated together in order to avoid frequent shell-to-shell collisions. These shells

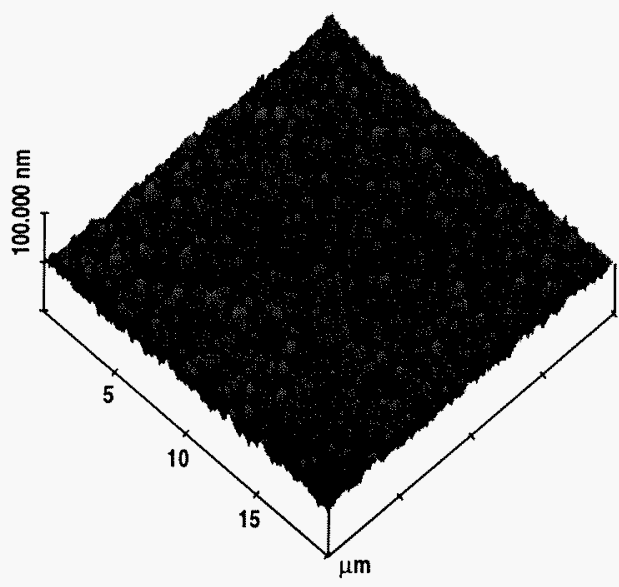

Fig. 2. AFM patch scan of a $100 \mu \mathrm{m}$ coating deposited on a freshly cleaved mica surface. The coating was homogeneous and contained virtually no isolated features. The RMS roughness (corresponding to the high mode region of Fig. 1) was $2.2 \mathrm{~nm}$. 
were coated with 100,150 and $200 \mu \mathrm{m}$ thicknesses. At each thickness approximately one-third of the shells were removed for measurement. The power spectra of a $200 \mu \mathrm{m}$ GDP shell along with that of the PAMS mandrel used for the coating are shown in Fig. 3. These shells meet the NIF standard indicating that the GDP coating process is capable of making NIF quality shells if proper PAMS mandrels are used.

Since initially only $2 \mathrm{~mm}$ diameter PAA-PAMS were available, this type of shells were used for fabrication of larger diameter GDP shells. These shells were coated using the tapping agitation system to avoid or minimize possible defect formation due to the agitation process. Although, the PAMS mandrels satisfied the NIF standard, the GDP shells were consistently much worse than the NIF standard. This was entirely unexpected given the experiments on $1 \mathrm{~mm}$ shells and the quality of these PAMS shells as measured by the spheremapper. However, closer examination of the PAA-PAMS power spectra compared to that of PVA-PAMS shells revealed that the background surface finish of PAA-PAMS was much worse than PVA-PAMS. This was confirmed by AFM patch scans as well. ${ }^{7}$ To further test the mandrel type effect on the final GDP shell in this case, a number of shells from each type were coated together in the same coating run. The two different types could be identified after having been mixed in the coating step because they had slightly different diameters. The spheremapper traces and power spectra for typical $55 \mu \mathrm{m}$ GDP shells from the two different batches are shown in Fig. 4. As can be clearly seen in the traces, shells made using the PAAPAMS are much rougher than those made using PVAPAMS. The power spectrum of the PVA-PAMS shell is much superior to that of the PAA-PAMS shell, although it is adversely affected by the presence of a single spike in the second set of traces. These types of isolated features on otherwise very smooth shells are discussed in more detail below. This and further experiments examining GDP coatings using PAA-PAMS showed the need for changing the PAMS fabrication process.

The remainder of the study involved various batches of $2 \mathrm{~mm}$ diameter PVA-PAMS. As noted above, part of our study involved making relatively thin walled $(\approx 14 \mu \mathrm{m})$ GDP shells to be used as mandrels in the polyimide coating development. The power spectra of a number of the best such shells are shown in Fig. 5. The best shells had power spectra that were below the NIF standard. More details on this work can be found in Ref. 7 . The other part of the study involved making thicker shells. We made shells at various thicknesses to examine the effect of increasing thickness on the quality of shells. Figure 6 shows the power spectra of 30 and $47 \mu \mathrm{m}$ GDP shells. Comparing these spectra to each other and those of $14 \mu \mathrm{m}$ shells shown in Fig. 5 indicates no major effect from the coating process. Finally, Fig. 7 shows the power spectrum of a $2 \mathrm{~mm}$ diameter, $125 \mu \mathrm{m}$ thick shell. The PVA-PAMS mandrels used for this coating had a mode $\sim 3$ defect that is reproduced as expected in the GDP shell. As can be seen, except for this defect the rest of the power spectrum lies at or beneath that required for NIF capsules. This is a clear demonstration that using suitable NIF size PAMS mandrels, GDP mandrels meeting the NIF criteria can be made.

Although, as shown above, shells meeting the NIF standard can be made, presently, the major remaining problem is the presence of a small number of isolated features on the final GDP shells. Figure 8 shows an example of the deleterious effect that such an isolated feature can have on the power spectrum of an otherwise
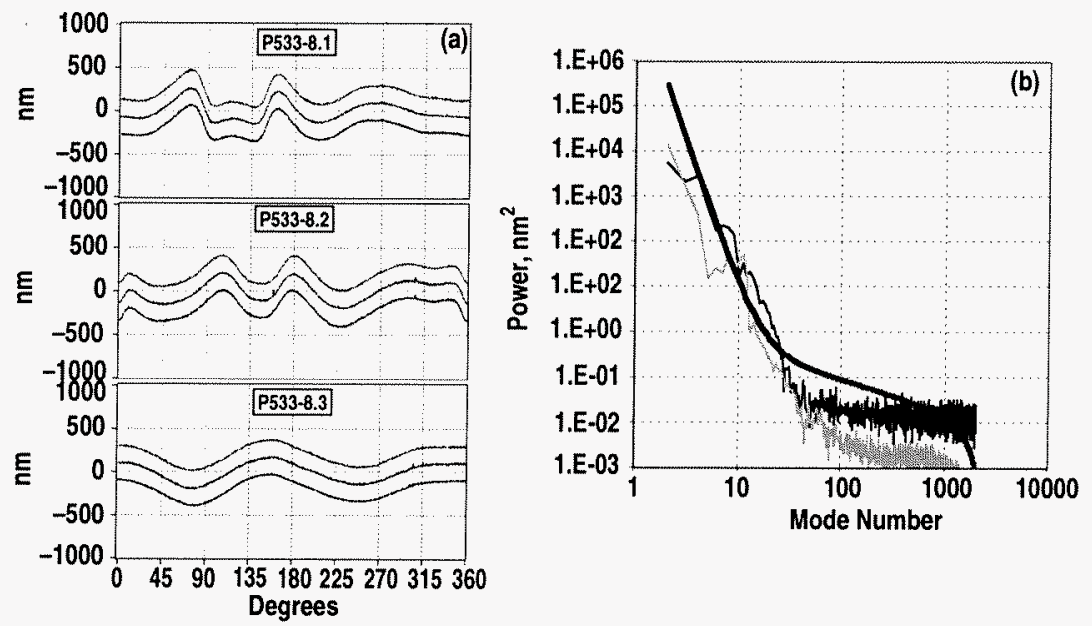

Fig. 3. Power spectrum of a $200 \mu \mathrm{m}$ thick, $1 \mathrm{~mm}$ diameter shell. This indicates that thick walled GDP shells suitable for NIF can in principle be made using the GDP process. 

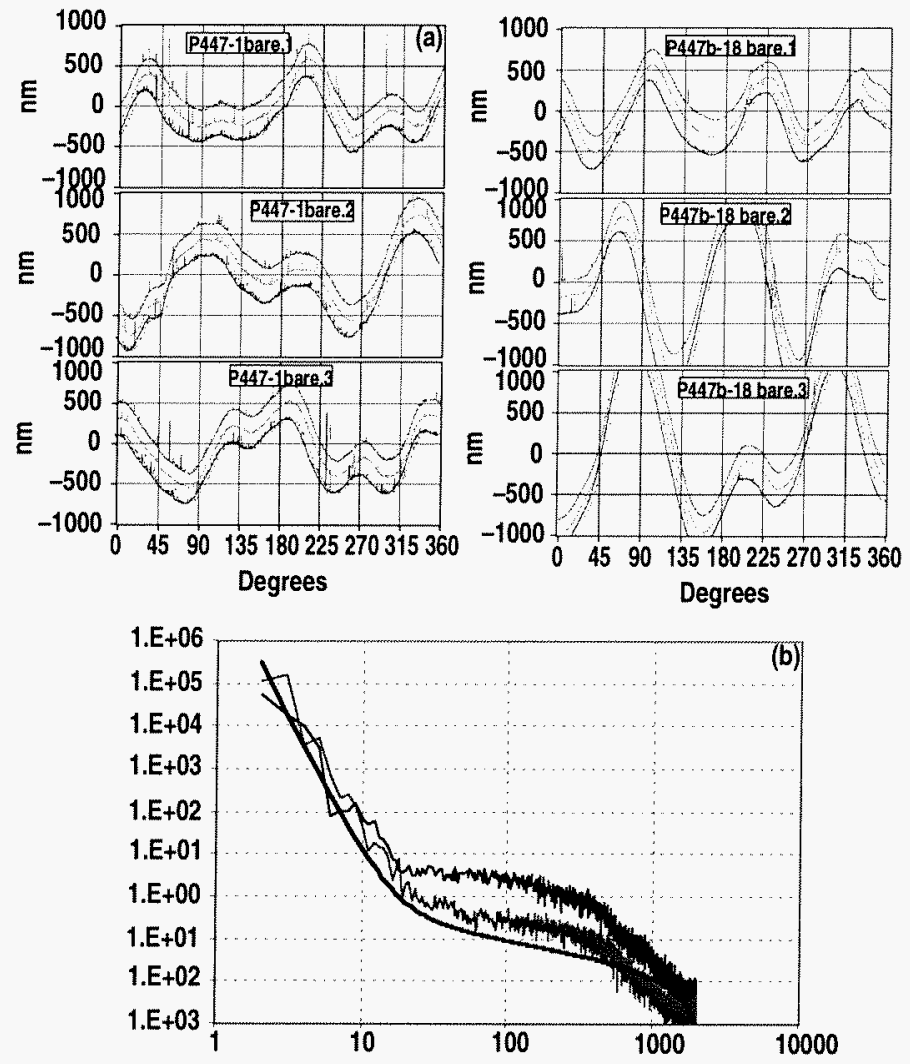

Fig. 4. The traces (a), and power spectra (b), of two shells from two different PAMS batches, one a PAA-PAMS and the other a PVAPAMS batch, made in the same GDP coating run. The traces of the GDP shell using the PAA-PAMS are much rougher than the one made using the PVA-PAMS, illustrating the effect of a poor PAMS mandrel on the final GDP shell. Note the isolated spike in the PVA-PAMS trace, which is the sole cause of the increase power in the GDP shell for modes $\rightarrow 100$.

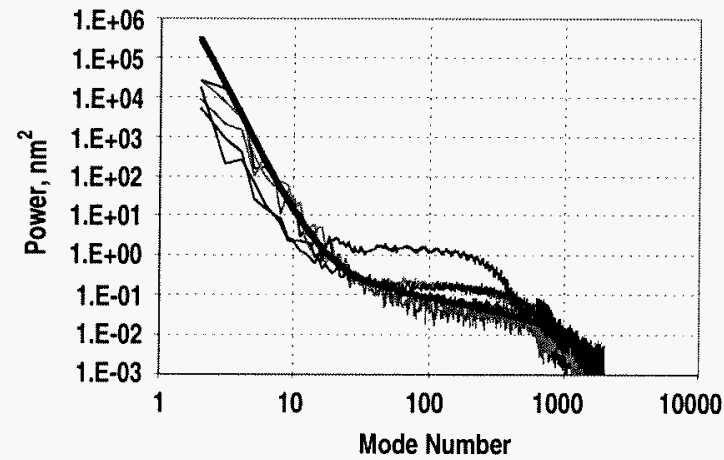

Fig. 5. The power spectra of $14 \mu \mathrm{m}$ mandrels made for further overcoating in the polyimide process. The variation from trace to trace is the result of presence of isolated spikes, caused by detection of domes in the traces of the various shells.

high quality $150 \mu \mathrm{m}$ thick, $1 \mathrm{~mm}$ diameter shell. The exclusion of the trace containing the isolated feature results in a power spectrum that is better than the NIF standard. This is similar to the example shown in Fig. 4 above for a $55 \mu \mathrm{m}$ GDP shell made using the PVA-PAMS mandrels. These isolated features can be divided into two categories. The first are large scale (hundreds of microns),

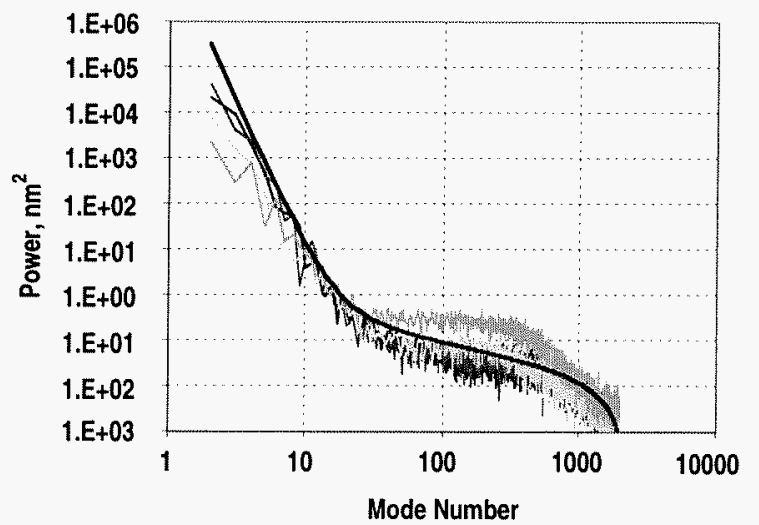

Fig. 6. Power spectra of 30 and $47 \mu \mathrm{m}$ GDP shells. The power spectra are similar to each other and to those shown in Fig. 5 for thinner shells.

low mode defects. These present themselves as shallow isolated depressions or lumps and are entirely associated with the PAMS mandrels. The other type which is more of a concern for the coating process, is the presence of very small scale (sub-micron to a few microns), high mode isolated features, commonly in the form of nearly hemispherical domes. These small features can act as 

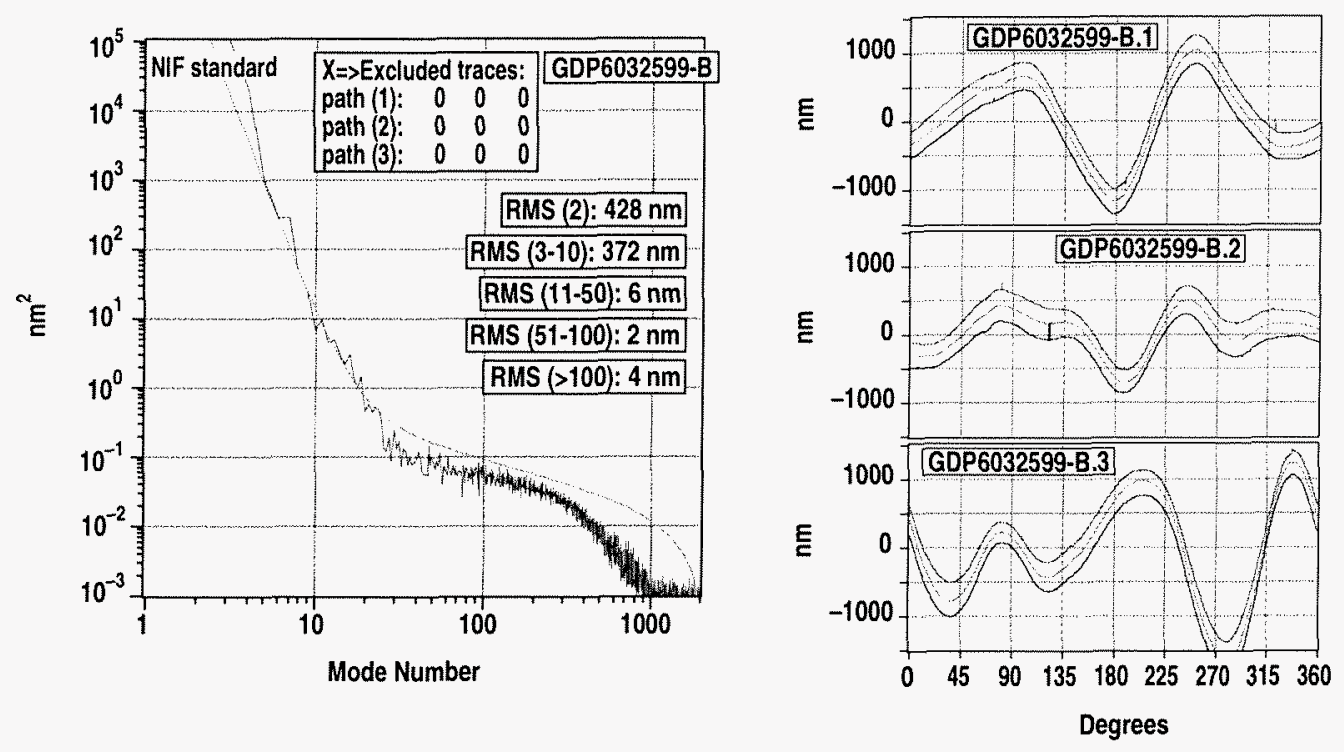

Fig. 7. Power spectrum of the best thick walled $(>100 \mu \mathrm{m}), 2 \mathrm{~mm}$ shells made to date. The shell thickness was $125 \mu \mathrm{m}$. The PAMS mandrel was made using the PVA process (see text).
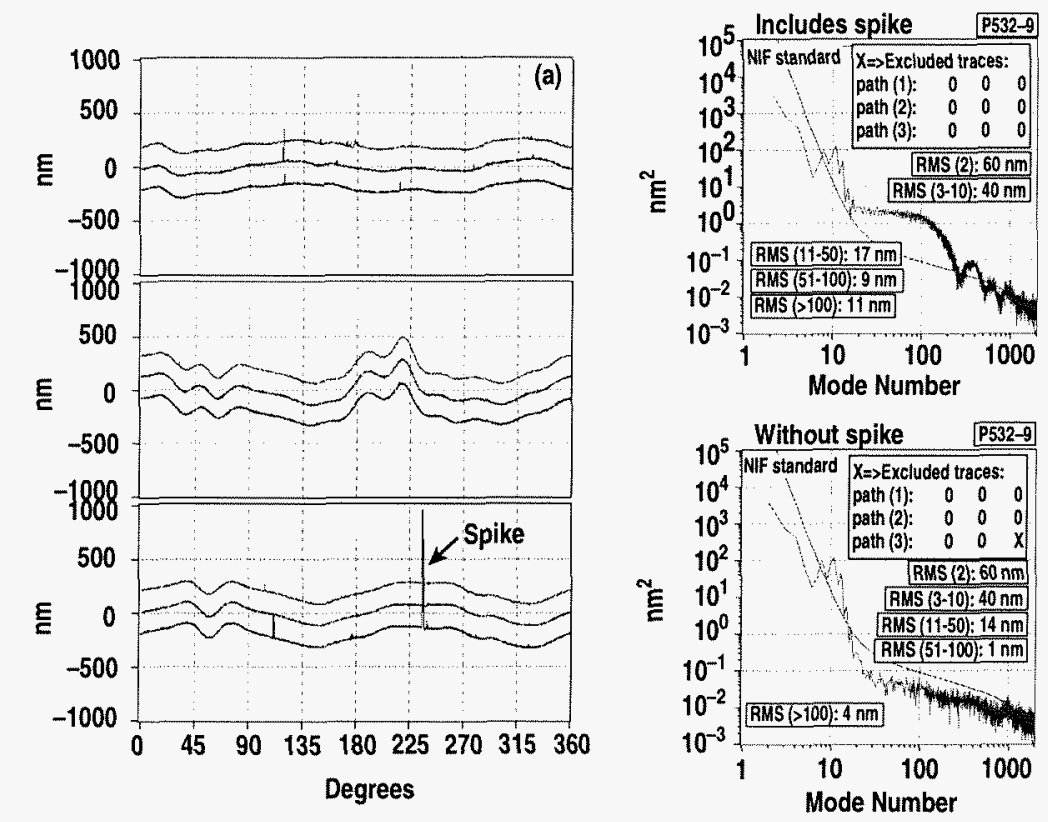

Fig. 8. The effect of a single isolated feature on the power spectrum of an otherwise very smooth shell. The power spectrum obtained by not including the trace containing the spike in the power spectrum calculation is below the NIF curve.

seeds for domes in the GDP coating when the PAMS mandrel is overcoated. Such domes grow in diameter during the GDP coating process and become more likely to be detected on the GDP shell by the spheremapping process.

There are a number of sources for such seeds for domes. The seeds may be present on the original PAMS shell. Small isolated imperfections can indeed be found in the spheremap traces of the starting PAMS shells. The imperfection may be debris or a protrusion present on the PAMS shell. If the seeds for the domes were protrusions in the surface of the shell, then the domes would be impossible to avoid in the GDP shells. The PAMS mandrel development effort is currently addressing this issue. An example of the effect of poor PAMS mandrels on the final GDP shells can be found in Ref. 8, which reports on fabrication of thick walled $(>120 \mu \mathrm{m})$ GDP shells in two different coaters, in two different countries. 
Another source for seeds for domes is loose debris attached to the shell. Debris may become attached to the shells in a number of steps in the shell fabrication procedure. Debris may be present on the shells immediately after manufacture. It can also become attached to the PAMS shell during handling before coating. If the GDP shell is to be further overcoated after pyrolysis, the debris may be attached to the shell during the pyrolysis step. Careful handling can in principal solve such problems.

The other source for seeds for domes is the coating process itself. The number of domes produced during the coating itself is indeed a major question remaining regarding the coating process. Careful examination of thick GDP coatings on mica substrates has shown virtually dome free surfaces, indicating virtually no dome production from the coating process itself. However, it has been previously shown that shell agitation can be a source of dome production during GDP coatings of $2 \mathrm{~mm}$ shells. 6 The same study also showed that coating fewer shells in any given run and gentle agitation, such as tapping, greatly minimizes dome production on shells. This is exactly the procedure that was used in fabrication of all the shells discussed in this paper. However, isolated feature can nonetheless be found on a large percentage of shells. Of course, the PAMS shells also contain such features as mentioned above. If the coating process were a major contributor to presence of domes on shells, then a significant increase in the number of spikes in the spheremapper traces should be observed. There would be a concomitant deterioration of power spectra as the shell thickness increased. The power spectra of shells shown in Figs. 5-7 for different GDP shells of increasing thickness do not vary significantly from each other. This is an indication that coating induced dome production is indeed not a major source of domes. This, however, does not rule out dome production at a low rate during the coating process. This will be the subject on future investigations in this area.

Due to the relatively small area probed by the spheremapper, detection of small $(\sim 20 \mu \mathrm{m})$ isolated features such as domes occurs only by chance. If these features are sufficiently tall $(\sim 1 \mu \mathrm{m})$, detection of a single feature increases the high mode power in the modal spectrum to above the NIF standard. Implosions simulations currently address homogeneous perturbations of various modes on the shell surface rather than isolated features. Therefore, it is not clear whether the presence a few high mode $(\approx$ mode 100$)$ isolated features truly does have an adverse effect on the implosion as it does on the modal power spectrum. Hence, more computational data is also desirable in determining an acceptable level of shell surface contamination by these features.

\section{CONCLUSIONS}

In this study we have shown the feasibility of fabricating GDP NIF scale capsules nearly meeting the current design surface finish requirements using the depolymerizable mandrel technique. The surface finish of the final GDP shells depends on that of the starting PAMS shell. When using the best PAMS mandrels, isolated defects are currently the major problem remaining in NIF scale GDP shell fabrication. While isolated lower mode defects are mainly a PAMS shell fabrication issue, elimination or reduction of higher mode defects involves the GDP mandrel fabrication process as well. Isolated higher mode features are observed on the starting PAMS shells as well as the final GDP shells. We have made shells at increasing GDP thicknesses and have observed no major increase in the number of the high mode isolated features. These shells of varying thicknesses have power spectra that are very similar to each other, indicating little deterioration of shell surface finish due to the GDP coating and shell agitation processes. Further implosion simulation data involving isolated features is needed to validate a major effort on complete elimination of high mode isolated features.

\section{ACKNOWLEDGMENT}

Work supported by the U.S. Department of Energy under Contract DE-AC03-01SF22260.

\section{REFERENCES}

1. S.A. Letts, et al., "Fabrication of Polymer Shells Using a Depolymerizable Mandrel," Fusion Technol. 28, 1797 (1995).

2. A. Nikroo, et al., "Capsule Development and Production for Inertial Confinement Fusion Experiments," Proc. of the First Conference on Inertial Fusion Sciences and Applications 1999, edited by C. Labaune, W.J. Hagan, K.A. Tanaka (Elsevier Press, Paris, 2000) 917.

3. S.W. Ferguson, et al., "Impedence-Power Effects on Plasma Polymer Surface Finish Using a Helical Resonator Discharge," J. Appl. Polym. Sci. 54, 107 (1994).

4. M. Takagi, et al., "Decreasing Out-of-Round in PolyAlpha-Methylstyrene Mandrels by Increasing Interfacial Tension,"Fusion Technol., 38, 46 (2000).

5. A. Nikroo, D. Woodhouse, "Bounce Coating Induced Domes on Glow Discharge Polymer Coated Shells," Fusion Technol. 35, 202 (1999).

6. McEachren, C.E. Moore, R.J. Wallace, "The Design, Performance, and Application of an Atomic Force Microscope-Based Profilometer," J. Vac. Sci. Tech. A 13, 983 (1995).

7. M. Takagi, R. Cook, et al. "Development of High Quality PAMS Mandrels for NIF," these proceedings.

8. M. Theobald, O.Legaie, P. Baclet and A. Nikroo, "Thick GDP Microshells for LIL and LMJ Targets," these proceedings. 